\title{
Diabetic nephropathy in pregnancy: new insights from a retrospective cohort study
}

\author{
Elisabeth R. Mathiesen
}

Received: 9 January 2015 / Accepted: 2 February 2015 / Published online: 18 February 2015

(C) Springer-Verlag Berlin Heidelberg 2015

Keywords Antihypertensive treatment - Blood pressure . Diabetic nephropathy $\cdot$ Perinatal mortality $\cdot$ Pregnancy . Preterm delivery

In this issue of Diabetologia, Klemetti and colleagues [1] present the results of the largest evaluation of clinical care in pregnant women with type 1 diabetes and diabetic nephropathy ever. This paper adds knowledge about the outcome, in present time, of these diabetic patients. The study is population based and covers more than 100 pregnant women with type 1 diabetes and nephropathy. A careful and proper evaluation of patient records was conducted, and the paper comes from a well-known centre with a long tradition for research and development within the field of diabetes and pregnancy.

The good news is that the perinatal mortality rate is reported to be $3 \%$ for these patients. This rate is comparable with that for diabetic patients without kidney involvement [2]. Advancements in ultrasound fetal flow measurements may have contributed to the prevention of stillbirths, and the intensification of the care of preterm infants may have contributed to the prevention of early neonatal deaths. The chance of women with diabetic nephropathy having a 'take home baby' is thus very high. Other studies have demonstrated that the risk of pregnancy-induced deterioration of diabetic nephropathy is low if women enter the pregnancy with a serum creatinine level $<200 \mu \mathrm{mol} / 1$ [3], and only one woman received dialysis treatment in the present cohort of pregnant women [1].

E. R. Mathiesen $(\bowtie)$

Center for Pregnant Women with Diabetes, Department of Endocrinology, 7551, Rigshospitalet, Faculty of Medicine,

University of Copenhagen, Copenhagen, Denmark

e-mail: em@rh.dk
This paper provides further evidence that intensive antihypertensive treatment during pregnancy is probably beneficial in these vulnerable women. Preterm delivery before 32 weeks was seen in $21 \%$ of the cases in this cohort during 2000-2011, and preterm delivery before 37 weeks was very common $(>70 \%)$, mainly due to severe hypertensive disorders. This leaves room for improvement. The majority of women in the present cohort did not receive antihypertensive treatment in early pregnancy. This strategy is often appropriate in women with mild essential hypertension, and is recommended in many international guidelines for antihypertensive treatment in pregnancy. The main reasons for this recommendation are that antihypertensive treatment may induce fetal growth restriction and that it is desirable to limit the use of medications that pass the placenta to the fetus. However, this may not be an effective approach in women with severe hypertension and kidney involvement, such as women with diabetic nephropathy. Blood pressure was within the target of $130 / 80 \mathrm{mmHg}$ in only $39 \%$ of the cohort in early pregnancy, and a high proportion developed severe hypertension with a high level of nephrotic proteinuria in late pregnancy.

In logistic regression analysis, first trimester blood pressure above $130 / 80 \mathrm{mmHg}$ and the last $\mathrm{HbA}_{1 \mathrm{c}}$ before delivery were associated with preterm birth before 37 gestational weeks. Furthermore, total urine protein excretion in the second and third trimesters correlated negatively with gestational age at birth. This paper thus supports the notion that in addition to the impact of poor glycaemic control uncontrolled hypertension might also contribute to the high prevalence of preterm delivery in these women.

In a cross-sectional study, women with diabetic nephropathy who had suboptimal control of blood pressure had an increased risk of preterm delivery compared with those with well-controlled blood pressure [4].

Our group in Copenhagen has published data on several small population-based series of pregnant women with kidney 
involvement (such as microalbuminuria or diabetic nephropathy) who were given early and intensive antihypertensive treatment, mainly methyldopa and labetalol, for blood pressure reduction [5-7]. The treatment goal was a blood pressure below $135 / 85 \mathrm{mmHg}$ and urinary albumin excretion below $300 \mathrm{mg} / 24 \mathrm{~h}$. If the woman was receiving antihypertensive treatment prior to pregnancy, the treatment was shifted to pregnancy-friendly drugs such as methyldopa without interruption in antihypertensive treatment. In our most recent publication, average blood pressure in both early and late pregnancy were approximately $128 / 70 \mathrm{mmHg}$, both in women with microalbuminuria and in those with diabetic nephropathy, and only one out of 41 women with kidney involvement developed nephrotic proteinuria [7]. The study also included women with type 2 diabetes, and women with kidney involvement showed similar pregnancy outcomes, regardless of type of diabetes. In women with type 1 diabetes and nephropathy from the two cohorts, one out of seven and three out of 11 , respectively $[6,7]$ delivered before week 34 , giving a prevalence of early preterm delivery of $22 \%$ in population-based samples from Copenhagen. Whether this reflects a better outcome than the prevalence of preterm delivery before week 32 in the study of Klemetti et al [1] is an open question, but prevention of severe hypertension and severe nephrotic proteinuria is probably beneficial for the mother. Thus, the tight blood pressure target aimed for in the Copenhagen study might be beneficial, but the number of pregnancies studied to date is too small to provide firm conclusions.

The use of low-dose aspirin to reduce the risk of preeclampsia, which is now generally recommended, was rarely given to the cohort studied by Klemetti and colleagues. Randomised controlled trials have suggested that the use of low-dose aspirin from early pregnancy might reduce the risk of pre-eclampsia in individually with a high risk of preeclampsia, including patients with diabetes [4].

Randomised controlled clinical trials investigating the effect of intensive antihypertensive treatment on the prevalence of pre-term delivery and the health of the newborn will probably not be performed in the future in this rare patient population with diabetic nephropathy.

Therefore, based on the findings of Klemetti et al [1] and other literature, it appears to be advisable to perform baseline assessments of proteinuria and kidney function before or in early pregnancy in women with diabetes to identify those in need of strict antihypertensive control.
In addition to aiming to achieve tight glycaemic and blood pressure control, low-dose aspirin treatment during pregnancy has been recommended in women with pregestational diabetes to reduce the incidence of pre-eclampsia and associated adverse maternal and neonatal outcomes.

The paper by Klemetti et al is thus a good example of how retrospective observational studies can help us to identify the right treatment for patients with rare diseases. It was only possible through the collection of data by a strong research group that for decades has focused on pregnancy outcomes in women with diabetes, coupled with the extraction of data by researchers from medical records kept over a period of more than 20 years.

Duality of interest The author declares that there is no duality of interest associated with this manuscript.

Contribution statement The author was the sole contributor to this paper.

\section{References}

1. Klemetti MM, Laivuori H, Tikkanen M, Nuutila M, Hiilesmaa V, Teramo K (2015) Obstetric and perinatal outcome in type 1 diabetes patients with diabetic nephropathy during 1988-2011. Diabetologia doi:10.1007/s00125-014-3488-1

2. Colstrup M, Mathiesen ER, Damm P, Jensen DM, Ringholm L (2013) Pregnancy in women with type 1 diabetes: have the goals from St. Vincent declaration been met concerning foetal and neonatal complications? J Matern Fetal Neonatal Med 26:1682-1688

3. Mathiesen ER, Ringholm L, Feldt-Rasmussen B, Clausen P, Damm P (2012) Obstetric nephrology: pregnancy in women with diabetic nephropathy - the role of antihypertensive treatment. Clin J Am Soc Nephrol 7:2081-2088

4. Carr DB, Koontz GL, Gardella C et al (2006) Diabetic nephropathy in pregnancy: suboptimal control associated with preterm delivery. Am J Hypertens 19:513-519

5. Ekbom P, Damm P, Feldt-Rasmussen B, Feldt-Rasmussen U, Mølvig J, Mathiesen ER (2001) Pregnancy outcome in type 1 diabetic women with microalbuminuria. Diabetes Care 24:1739-1744

6. Nielsen LR, Damm P, Mathiesen ER (2009) Improved pregnancy outcome in type 1 diabetic women with microalbuminuria or diabetic nephropathy: effect of intensified antihypertensive therapy? Diabetes Care 32:38-44

7. Damm JA, Asbjörnsdóttir B, Callesen NF et al (2014) Diabetic nephropathy and microalbuminuria in pregnant women with type 1 and type 2 diabetes: prevalence, antihypertensive strategy, and pregnancy outcome. Diabetes Care 36:3489-3494 